\title{
2 Historische Ausgangslage
}

Als Ausgangslage, um das Bild der Nuntien über die Eidgenossenschaft zu verstehen und einzuordnen, ist das Weltbild ihrer „Auftraggeber“ ausschlaggebend. ${ }^{1}$ Die Päpste, die vom Ende des 16. bis zur Mitte des 17. Jahrhunderts wirkten, waren insgesamt zehn. Angefangen von Sixtus V. (1585-1590), ${ }^{2}$ der sich als „Reformer“ ${ }^{3}$ hervorhob, bis Innozenz X. (1644-1655), ${ }^{4}$ dem Papst, der zur Zeit des Westfälischen Friedens im Amt war. Es sind Päpste der posttridentinischen Zeit. ${ }^{5}$ Ausgehend von einer Einstellung, die eine Konsolidierung des päpstlichen Zentralismus anstrebte, kann man somit die „päpstliche Sicht“ auf die Eidgenossenschaft so verstehen, dass sie vor allem auf die „religiösen Feinde“ konzentriert war und andererseits auf die Durchsetzung der konziliaren Dekrete in den katholischen Gebieten. Auch wenn keiner der Päpste in dieser Zeit mit dem Vorgänger Julius II. in militärischer Hinsicht zu vergleichen ist, so galt in dieser Epoche doch das Söldnerwesen als eines der wichtigsten Interessenfelder der Päpste in Bezug auf das Verhältnis zur Schweiz. Die Päpste, die wir hier genauer betrachten wollen, hatten nicht nur die Stärkung der eigenen Truppen im Auge, sondern auch die militärische und politische Unterstützung „alliierter katholischer Kräfte" wie Frankreich und Spanien. ${ }^{6}$

Was die Bedeutung des Dreißigjährigen Kriegs auf dem Gebiet der Eidgenossenschaft betrifft, so war die Schweiz davon wenig betroffen und wenn, dann nur indirekt. Das lag auch daran, wie Zurfluh in seinem Beitrag für das Historische Lexikon der Schweiz festhält, dass „die demografische und wirtschaftliche Vormachtstellung der reformierten Städteorte [...] politisch nicht

1 Vgl. Aaron J. Gurjewitsch: Das Weltbild des mittelalterlichen Menschen, S. 160: „Die Unfähigkeit des mittelalterlichen Menschen, Welt und Gesellschaft in ihrer Entwicklung zu sehen, ist die Kehrseite seiner Einstellung zu sich selbst und zu seiner inneren Welt. Das Mitglied einer Gruppe, der Träger einer ihm übertragenen Funktion oder Dienststellung, das Individuum strebte vor allem danach, einem vorgeprägten Typ maximal zu entsprechen und seine Pflicht gegenüber Gott zu erfüllen.“

2 Vgl. Silvano Giordano: Sisto V.

3 Vgl. Peter Stephan: Transformation und Transfiguration.

4 Vgl. Guido Braun: Innozenz X.

5 Vgl. Daniel Büchel, Volker Reinhardt (Hrsg.): Modell Rom?, S. 274: „Durch die in Rom im Verhältnis zu anderen europäischen Höfen hervorstechenden Defizite an Patronatssicherheit und damit Laufbahnplanbarkeit aber dürfte sich nicht nur ein System von sozialen Netzen und doppelten Böden, sondern auch, je länger desto stärker, eine zumindest idealtypisch definierbare ,Kurialmentalität‘ herausgebildet haben - mit zentralen Leitwerten sowie Phobien und Feindbildern.“

6 Vgl. Martin Papenheim: Machen Päpste Politik?

ə Open Access. (C) 2021 Mario Galgano, publiziert von De Gruyter. $(\leftrightarrow)$ Er Dieses Werk ist lizenziert unter der Creative Commons Attribution 4.0 Lizenz. Weitere Informationen finden Sie unter 
zum Tragen" kam. ${ }^{7}$ Da die katholischen Orte mit Spanien und Österreich verbündet waren und in der Tagsatzung die Mehrheit bildeten, kam es zu diesem Zustand des „Ausgleichs“. Es wundert nicht, dass die Nuntien versuchten, die Stellung der katholischen Parteien - sowohl in der Schweiz als auch auf europäischer Ebene - zu schützen, zu stärken oder wenn möglich zur dominierenden Kraft zu fördern. Die verschiedenen und komplexen Bündnisse führten aber dazu, dass die Handlungsfreiheit und Fähigkeit zu dominieren der entsprechenden Orte und Parteien sehr eingeschränkt wurde. Die Nuntien mussten sich somit mit der Kompromiss- und Neutralitätspolitik der Eidgenossen zufriedengeben. ${ }^{8}$

Die konfessionelle Spaltung der Eidgenossen prägte die Struktur derer Außenbeziehungen. Seit dem frühen 17. Jahrhundert hatten alle dreizehn Kantone das Bündnis mit Frankreich von 1521 zugestimmt. Es gab aber daneben noch ein exklusives katholisches Bündnis mit Savoyen seit 1560 und mit SpanienMailand ab 1587. Die Entsendung der entsprechenden Gesandten - zusammen mit dem Nuntius aus Rom - können also auch unter diesem Aspekt betrachtet werden: die katholische Schweiz war mit den katholischen Groß- und Regionalmächten verbunden. Auf der anderen Seite gab es auch Bündnisse unter protestantischer Orte. Hier waren Zürich und Bern die führenden Orte, die beispielsweise 1588 mit Straßburg ein Bündnis schlossen. Die protestantische Schweiz pflegte ab 1615 auch intensive diplomatische Beziehungen zu Venedig. Die „Serenissima“ war in jener Zeit auf diplomatischer Ebene eine scharfe Konkurrenz zum Kirchenstaat. ${ }^{9}$

Auf der anderen Seite verzichteten die Eidgenossen auf ständige Vertretungen an europäischen Höfen. Das lag wohl daran, dass sie einerseits keine einheitliche „Außenpolitik“ hatten und andererseits auch nicht die damals üblichen adeligen Diplomaten, die sich mit dem Zeremoniell auseinandersetzen konnten, da eine ständische Qualifikation fehlte. ${ }^{10}$ Es war im Übrigen im 16. und 17. Jahrhundert nicht immer üblich, gegenseitig Gesandten zu schicken. Ein dritter Punkt für die fehlende Schweizer Gesandten in Europa waren auch die hohen Kosten, die sich die Eidgenossen nicht leisten konnten oder wollten. ${ }^{11}$

7 Zit. nach: Anselm Zurfluh: Der Dreissigjährige Krieg, in: Historisches Lexikon der Schweiz (online): https://hls-dhs-dss.ch/de/articles/008907/2015-03-05/ (31. Dezember 2020).

8 Vgl. Ralph Rotte: Die Außen- und Friedenspolitik des Heiligen Stuhls.

9 Vgl. Andreas Würgler: Individuelle und kollektive Akteure der Alten Eidgenossenschaft, S. 81.

10 Wie Würgler hervorhebt, „begnügten sich“ die Eidgenossen „mit informellen Agenten“. Diese seien in Paris, Mailand, Madrid und Rom gewesen. Vgl. Ebd.

11 Vgl. Liisi Karttunen: Les nonciatures apostoliques permanentes de 1650 à 1800 . 


\subsection{Die Päpste zwischen 1586 und 1654}

Zur Übersicht der Päpste hilft eine Auflistung der entsprechenden Kirchenoberhäuptern ${ }^{12}$ :

1. Sixtus V. (Felice Peretti di Montalto): 1585-1590 (Drastische Reformen: hohe Strafen für Ehebruch und verschiedene Vergehen, dadurch hohe Einnahmen, am Ende einer der reichsten Herrscher Europas)

2. Urban VII. (Giovanni Battista Castagna): 1590 (Starb vor der Krönung an Malaria)

3. Gregor XIV. (Niccolò Sfondrati): 1590-1591

4. Innozenz IX. (Giovanni Antonio Facchinetti): 1591

5. Clemens VIII. (Ippolito Aldobrandini): 1592-1605 (Ließ 1600 Giordano Bruno hinrichten)

6. Leo XI. (Alessandro Ottaviano de' Medici): 1605

7. Paul V. (Camillo Borghese): 1605-1621

8. Gregor XV. (Alessandro Ludovisi): 1621-1623

9. Urban VIII. (Maffeo Barberini): 1623-1644 (Galileo Galilei wird verurteilt)

10. Innozenz X. (Giovanni Battista Pamphilj): 1644-1655 (Westfälischer Friede 1648)

Um jene Päpste besser einzuordnen, ist es unabdingbar, auch die Bedeutung des Konzils von Trient (1545-1563) zu berücksichtigen. Seine Auswirkungen sind bis heute in der katholischen Kirche zu spüren. ${ }^{13}$ Dabei war der im Zuge des Konzils von Trient festgelegte Messritus über vier Jahrhunderte die Norm für die römisch-katholische Kirche. Als Papst Paul III. am 19. November 1544 mit der Bulle „Laetare Jerusalem“ („Freue dich, Jerusalem“) das Konzil einberief, war nicht Rom das Zentrum der Kirche, sondern die norditalienische Stadt Trient. Mit dem sogenannten Tridentinum leitete die katholische Kirche nach der Reformation ihrerseits eine grundlegende Erneuerung ein. Doch im Nachhinein betrachtet kam das Konzil für eine Überwindung der Kirchenspaltung, die sich seit 1517 durch zahlreiche gegenseitige Konfrontationen gefestigt hatte, einfach zu spät. Das lag wohl auch daran, dass die Beratungen nicht optimal verliefen, zumindest nicht so, wie es die Initiatoren sich erhofft hatten. Es muss-

12 Vgl. Rudolf Lill: Die Macht der Päpste, Kevelaer 2011.

13 Man denke an den Streit um die sogenannte lefevbrianische Piusbruderschaft, die an der „Tridentinischen Messe“ festhält, was wiederum andere als „etwas Gestriges“ kritisieren. Das liegt daran, dass der Priester mit dem Rücken zur Gemeinde zelebriert. Der Gottesdienst wird hierbei ausschließlich auf Latein gefeiert, was bei lateinunkundigen Gläubigen dazu führte, dass sie die Wandlungsworte des Priesters als „Hokuspokus“ missverstanden (von „hoc est enim corpus meum“, „dies ist mein Leib“). 
te schon damals als wenig rühmlich erscheinen, dass man 18 Jahre brauchte, um zu einem Abschluss zu kommen. Mit diesem Zeitraum ist das Konzil von Trient eines der längsten überhaupt. ${ }^{14}$

Seine Voraussetzungen waren sehr unterschiedlich, denn seit längerer Zeit war ein Konzil im Rahmen der Aufarbeitung der protestantischen Lehren dringend gefordert worden. Es gab aber lange Zeit einen, der sich dagegen stemmte, und das war kein geringerer als der Papst selber - in diesem Fall Clemens VII. (1523-1534) -, der, so gut es ging, zu einem Aufschub drängte. Er fürchtete den Konziliarismus wie der Teufel das Weihwasser. ${ }^{15}$

Zum Tagungsort ist festzustellen, dass mit Trient eine Stadt weit weg von Rom gewählt wurde. Sie gehörte zum Reich und dennoch wurde das Konzil als „römische Angelegenheit“ betrachtet. Ein „papstfreies Konzil“, wie es die Protestanten für eine Teilnahme ihrerseits gefordert hatten, gab es nicht - im Gegenteil: Paul III. (1534-1549) stellte die Versammlung ausdrücklich unter seine Leitung. ${ }^{16}$

Das Fremde und die eigene Identität - dieser Gegensatz schlug sich auch in der Zahl der Teilnehmer nieder. Es waren nur 31 Bischöfe überhaupt dabei und davon ausschließlich spanisch-kaiserliche und päpstliche aus Italien, die sich gegenseitig beargwöhnten. Das war zumindest 1545 zur Eröffnung der Fall. Die Teilnahme deutschsprachiger, direkt von der Reformation betroffener Konzilsväter in der zweiten Sitzungsperiode 1551/52 blieb nur eine Episode. Sie reisten ab, weil ihre Forderungen komplett durchfielen. Spannungen und Konflikte prägten das Konzil während seiner gesamten Dauer. Am Ende war es wohl nur dem Verhandlungsgeschick des letzten Konzilspräsidenten Giovanni Morone zu verdanken, dass die Versammlung zu einem guten Ende kam. Das ursprüngliche Ziel, eine Verständigung mit den Protestanten, geriet sehr bald in unerreichbare Ferne. Das Konzil konzentrierte sich darauf, theologische Antworten auf die protestantische Herausforderung zu geben und die katholischen Lehren festzuzurren. Die behandelten Themen hatten insofern die Protestanten gesetzt, etwa wenn - in Abgrenzung zum evangelischen „sola scriptura“ - Schrift und Tradition als die zwei Quellen der Offenbarung genannt wurden. ${ }^{17}$

Auch wenn der Umfang der Reformen begrenzt war, gab das Konzil dem Katholizismus über 300 Jahre sein Gepräge. Vieles wurde freilich erst nach vielen Jahrzehnten wirksam, so das dort formulierte Bischofsideal oder die Hebung

14 Vgl. Johann Georg Meyer: Das Konzil von Trient und die Gegenreformation in der Schweiz. 15 Ebd.

16 Vgl. Hubert Jedin: Papst und Konzil, S. 429-440.

17 Ebd. 
des Bildungsniveaus bei Klerikern. Der Zölibat wurde neu eingeschärft, Pfarrer wie Bischöfe und Kardinäle zum Wohnen vor Ort verpflichtet.

Theologisch ging es etwa um Heiligen- und Reliquienverehrung, Ablass, Fegefeuer oder um das Verständnis von Rechtfertigung, Gnade und Kirche. Die Siebenzahl der Sakramente - Taufe, Firmung, Buße, Eucharistie, Ehe, Priesterweihe und Krankensalbung - wurde festgelegt, die Führung ordentlicher Ehestandsregister vorgeschrieben. Für eine Reform brauchte die Kirche auch neue Methoden der Verkündigung und Mission. Der 1534 gegründete Jesuitenorden erwies sich dafür als ein modernes Instrument. ${ }^{18}$

Nicht alles wurde nach der Krise des Konzils 1562/63 am Ende fertig. Papst Pius V. veröffentlichte im Auftrag des Konzils den „Index der verbotenen Bücher“, 1564 das sogenannte Tridentinische Glaubensbekenntnis mit Gehorsamsversprechen gegenüber dem Papst, 1566 den Römischen Katechismus, 1568 das Priesterbrevier und 1570 das Römische Messbuch. ${ }^{19}$

Der wichtigste Erforscher des Tridentinums, Hubert Jedin ${ }^{20}$ (1900-1980), sah in dem Konzil den Antriebsimpuls zu einer inneren Erneuerung der Kirche: „Es gab der Theologie wie der Glaubensverkündigung klare Normen, es grenzte lehramtlich ab, aber es trennte nicht, wo nicht schon die Trennung war." ${ }^{21}$ Dabei habe es nicht einfach das Mittelalter restauriert; „es modernisierte Verfassung und Seelsorge“.22

\subsubsection{Von Sixtus V. zu Innozenz X.: Historische Entwicklung der Pontifikate}

In dieser Zeit kam es zu einer „erfolgreichen Durchsetzung“ der Machtposition der Päpste im europäischen Kontext, was dann fast drei Jahrhunderte lang die katholische Kirche prägte. ${ }^{23}$ Und diese Situation verdankten die Päpste nicht zuletzt den damals neu geschaffenen Instrumenten der Diplomatie ${ }^{24}$, genauer ge-

18 Vgl. Joseph Studhalter: Die Jesuiten in Luzern 1574-1652.

19 Vgl. Hubert Jedin: Papst und Konzil, S. 429-440.

20 Vgl. Hubert Jedin: Geschichte des Konzils von Trient. Es handelt sich um ein fünfbändiges Werk.

21 Zit. nach: Hubert Jedin: Papst und Konzil, S. 429-440.

22 Zit. nach: Hubert Jedin: Il tipo di vescovo secondo la riforma cattolica, Brescia 1950.

23 Vgl. Rudolf Lill: Die Macht der Päpste, S. 57.

24 Wie Scholtyseck und Freiberger schreiben, ist die Bedeteutung des Begriffs „Diplomatie“ einem „dauerhaften Wandlungsprozess“ unterworfen. Vgl. Joachim Scholtyseck, Thomas Freiberger: Diplomatie, S. 1101: „Der heutige Begriff von Diplomatie als System von Staatenbeziehungen mit ständigen Vertretungen, einem ausdifferenzierten Gesandtschaftswesen, einem Gesandtschaftsrecht und allgemeinverbindlichen Umgangsformen ist hingegen eine „Erfindung “ des frühneuzeitlichen Europas.“ 
sagt der Gründung von Nuntiaturen, die in Luzern schon 1578, ${ }^{25}$ in Graz 1580, in Wien 1581, in Köln 1584 und in Brüssel 1597 eingesetzt wurden. An all diese Außenposten wurden meist italienische Prälaten gesandt. ${ }^{26}$

Stellten frühere Werke von Historikern die Päpste des ausgehenden 16. und des 17. Jahrhunderts als „bedeutungslose Kirchenoberhäupter“27 dar, so ist die heutige Sichtweise der Geschichtswissenschaft differenzierter. Allein die barocke Kultur des 17. Jahrhunderts, die das Papsttum prägte, ist heute noch Sinnbild und Sinnträger für eine bestimmte Einstellung der Kirche - und wie wir die damalige Kirche betrachten. Doch was die Kunst und Kultur einerseits betrifft, muss andererseits nicht zwangsläufig auch den politischen Einfluss des Papsttums in jener Zeit an sich betreffen.

Wenn wir den Ausgangspunkt unserer Betrachtung auf 1586 legen, so sehen wir unter Papst Sixtus V. (1585-1590) die Fertigstellung der Kuppel des Petersdomes sowie die heute noch sichtbaren Kolonnaden Gian Lorenzo Berninis. Historiker sind sich einig, dass unter Sixtus V. und dann unter Alexander VII. (1655-1667) das Papsttum einen neuen Höhepunkt in Sachen Machtpolitik erreichte, auch wenn derselbe Alexander VII. von Ludwig XIV. von Frankreich aufs tiefste gedemütigt wurde. ${ }^{28}$ Diese Auseinandersetzung sowie auch die Wirren des Dreißigjährigen Krieges (1618-1648) prägten die Weltsicht der Päpste

25 Vgl. ebd., S. 59.

26 Vgl. Alexander Koller: Einige Bemerkungen zum Karriereverlauf der päpstlichen Nuntien am Kaiserhof (1559-1655), S. 841: ,... nach dem Studium (meist der Iurisprudenz) begann die Karriere in aller Regel als Sekretär, Abbreviator usw. in einer kurialen Behörde oder außerhalb der Kurie bei einem Nuntius oder Legaten. Entscheidend war dann, in den Besitz eines kurialen Schlüsselamtes zu gelangen, das den weiteren Aufstieg ermöglichte, also ein Referendariat, Auditoriat oder Protonotariat. Von dieser Position aus konnte bereits die Übernahme einer Nuntiatur erfolgen. Oftmals sammelten die künftigen Nuntien jedoch zuvor noch zusätzlich zu ihrer Tätigkeit in der Zentrale (an der Kurie) administrative Erfahrungen in den Provinzen des Kirchenstaats durch die Ausübung eines oder mehrerer governi. Nach Abschluß der Nuntiatur wartete auf einige Prälaten der Purpur als Krönung ihrer diplomatischen Laufbahn. Als Kardinäle übernahmen die ehemaligen Nuntien mitunter weitere diplomatische und administrative Aufgaben, sog. Legationen, und/oder übernahmen Funktionen an der Kurie. Von denen, die nicht Kardinäle wurden, erhielten die einen weitere diplomatische Aufgaben, für andere bedeutete das Ende der Nuntiatur auch das Ende der Karriere, was meist den Rückzug in die frühere Diözese bedeutete. Im besten Fall erhielten sie eine neue, einkunftsträchtigere Diözese, und wurden evtl. später nochmals für eine diplomatische Mission reaktiviert. Nicht zuletzt sollte auf den Tatbestand hingewiesen werden, dass eine nicht zu vernachlässigende Zahl während der Ausübung des Mandats starb.“

27 Als Beispiel: Leopold von Ranke: Die römischen Päpste. Bd. 1. Berlin 1834.

28 Dies gilt im Hinblick auf das nachtridentinische Papsttum. Bereits im 13. Jahrhundert wurde unter Papst Bonifaz VIII. (1294-1303) eine große Machtstellung erreicht. Bonifaz VIII. trieb den weltlichen Machtanspruch des Papsttums auf die Spitze. Vgl. dazu Leopold von Ranke. 
der damaligen Zeit. Dass die Rolle des Papstes in Europa unter Druck stand, kann man zweifelsohne sagen, doch das Bild eines Papsttums am Rande der Bedeutungslosigkeit, wie sie von protestantisch-liberalen Historikern verbreitet wurde, ${ }^{29}$ gilt heute nicht mehr. Vielmehr ist - wie so oft in der Geschichte der Menschheit - ein kompliziertes und facettenreiches Bild des Papsttums Ende des 16. bis Mitte des 17. Jahrhunderts gültig. ${ }^{30}$

Die Eidgenossenschaft spielte in zweierlei Hinsicht für die Päpste des 16. und 17. Jahrhunderts eine bedeutende Rolle: Einerseits war die Schweiz durch ihre geostrategische Lage wichtig für die Machtpolitik und somit von Interesse für das Kirchenoberhaupt in Rom, und andererseits ging es dem Papsttum in jener Zeit auch kirchenpolitisch darum, seine religiöse Rolle zu verteidigen und zwar vor allem gegen die aggressive Stellung der protestantischen Kräfte. Nicht zu vergessen ist, dass der Kirchenstaat unter der Führung des Papstes eine italienische Mittelmacht war und vor allem als Gegenpol zu Venedig zu sehen ist. ${ }^{31}$

Für das Papsttum sind die drei genannten Bereiche der Machtpolitik, Kirchenpolitik und Kirchenstaat nicht einfach voneinander $\mathrm{zu}$ trennen oder getrennt zu betrachten. Das Papsttum verstand sich - und wurde von außen auch so verstanden - als Medaille mit zwei Seiten: Auf der einen Seite galt der Papst als geistliches Oberhaupt der Kirche und auf der anderen als Weltherrscher zusammen oder sogar gegen den Kaiser und die Könige Europas. Damit verbunden ist die wirtschaftliche Dimension. Der Kirchenstaat brachte und brauchte Geld, und um eine angemessene Machtpolitik zu führen, bedurfte es ebenfalls finanzieller Mittel. Doch als Kirchenoberhaupt konnten die Päpste - und darauf pochten sie damals - auf die Finanzierung aus der Weltkirche rechnen. So war Sixtus V. nicht nur ein halbwegs erfolgreicher Machtpolitiker und der Reorganisator von Kirche und Kirchenstaat, sondern zugleich trotz oder gerade wegen seiner dubiosen Methoden ein nach den Begriffen der Zeit äußerst erfolgreicher Finanzpolitiker.

Um das römische Bild der Eidgenossenschaft zu verstehen, muss man auch das Ursprungsland der Nuntien und der Päpste im Blick haben. Da sie fast alle Italiener waren, soll die Lage Italiens in jener Zeit in den Blick genommen werden. So war das Land zwar bekanntlich noch nicht vereinheitlicht worden, und machtpolitisch war es ab 1530 - und vor allem ab 1559 - vorwiegend durch die spanische Vorherrschaft geprägt. Wir dürfen nicht vergessen, dass seit 1494 Spanien und Frankreich in Süd- wie in Norditalien heftig um Einfluss und Besitzungen rangen. Nach der kläglichen Niederlage des Spanienhassers Paul IV.

29 Vgl. dazu Leopold von Ranke.

30 Vgl. dazu Historiker wie Jean Delumeau.

31 Vgl. Hedwig Bach: Leitbild für die Reform der Kirche nach dem Konzil von Trient. 
(1555-1559), der noch einmal einen Krieg an der Seite Frankreichs gewagt hatte, mussten die Päpste sich mit Spanien arrangieren, während umgekehrt die spanische Krone daran interessiert blieb, dass der Stuhl Petri mit einem Freund Spaniens besetzt war. Denn zunächst blieb der Papst ein wichtiger Partner für die spanische Innen- und Außenpolitik. Unter anderem, weil beide nur auf der Grundlage der Besteuerung der spanischen Kirche funktionierten, die wichtiger war als alle Silberschätze Amerikas, und diese wiederum funktionierte am besten mit päpstlicher Hilfe. Zwar gelang es den spanischen Kardinälen und Diplomaten nur selten, bei der Papstwahl den Wunschkandidaten ihres Königs durchzusetzen, aber von den 16 zwischen 1559 und 1670 gewählten Päpsten waren die meisten bewährte Spanienfreunde oder zumindest für Spanien akzeptabel - schlimmstenfalls als das kleinere Übel wie der frankophile Leo XI. Medici (1605), denn der Alternativkandidat wäre der spanienkritische Kirchenhistoriker Baronius gewesen. Der frankreichfreundliche Urban VIII. (1623-1644) hingegen kam ohne Einwirkung der Großmächte auf den Thron. ${ }^{32}$

Seit der Mitte des 16. Jahrhunderts gestattete es die relative Stabilisierung der italienischen Verhältnisse den Päpsten, sich stärker als ihre Vorgänger über die Interessen des Kirchenstaats hinaus machtpolitisch zu engagieren. Neben der in der katholischen Welt nach wie vor gewichtigen politischen oder rechtlichen Parteinahme des Stellvertreters Christi als solcher konnte politisches Engagement die Entsendung päpstlicher Truppen bedeuten wie 1591 gegen die Hugenotten nach Frankreich oder 1595 gegen die Osmanen nach Ungarn. Daneben engagierten sich die Päpste finanziell entweder durch die Bewilligung von Sondersteuern auf die Kirche der Länder ihrer jeweiligen Verbündeten oder durch direkte Hilfsgelder, die von der Papstfinanz aufgebracht wurden. Noch im Dreißigjährigen Krieg wurden Zahlungen an den Kaiser und die Katholische Liga geleistet. Die großen Ziele der päpstlichen Politik waren dabei erstens wie schon im Spätmittelalter die Abwehr der Osmanen, zweitens die Gegenreformation als politische Bekämpfung des Protestantismus, drittens die Erhaltung oder gegebenenfalls die Wiederherstellung des Friedens zwischen den katholischen Mächten. ${ }^{33}$

Zur Diplomatie der Päpste im 15. und 16. Jahrhundert kommt hinzu, dass die sich verändernde Struktur des päpstlichen Legatenwesens zum allmählichen Schwinden der Kardinalslegationen führte, die durch den Einsatz von apostolischen Nuntii abgelöst wurden. ${ }^{34}$ Anfang des 16 . Jahrhunderts bildete

32 Vgl. BBKL.

33 Vgl. Johann Georg Meyer: Das Konzil von Trient und die Gegenreformation in der Schweiz. 34 Vgl. Joachim Scholtyseck, Thomas Freiberger: Diplomatie, S. 1101: „Bis ins Mittelalter hinein gab es keine ständigen diplomatischen Vertretungen, keine speziell mit Außenpolitik be- 
sich die Institution der permanenten päpstlichen Vertretung, der Nuntiaturen an den Herrscherhöfen Europas heraus - Ergebnis und Indiz einer voranschreitenden Klerikalisierung und Bürokratisierung des kurialen Gesandtschaftswesens. Da die Kardinäle selbst großes Interesse daran hatten, in Rom zu bleiben, wandelte sich das Entsenden von Kardinallegaten in die Reiche nördlich der Alpen in der zweiten Hälfte des 15. Jahrhunderts bisweilen zu einer päpstlichen Strafmaßnahme, beziehungsweise wurde bei unliebsamen oder zu mächtigen Kardinälen eingesetzt, um diese vom päpstlichen Hof zu entfernen. ${ }^{35}$

\subsubsection{Die Schweiz-Bezüge der einzelnen Pontifikate}

Durch den Gardekommandant ${ }^{36}$ Jost Sägisser ließ Luzern den frisch ernannten Papst Sixtus V. ein Gratulationsschreiben überreichen, was mit einem Dankesbrief aus Rom beantwortet wurde. ${ }^{37}$ Dem Papst war es ein Anliegen, mit der Eidgenossenschaft nicht nur ein „militärisches Bündnis“ durch die Anwerbung neuer Schweizergardisten zu fördern. Wie Balthasar aufzählt, hatte Sixtus V. „die Kirchenzucht, die in dieser Republik wegen der Nähe des ketzerischen Giftes sehr zerfallen und angesteckt war“, ${ }^{38}$ dazu gebracht, einen Nuntius nach Luzern zu entsenden. Doch nicht nur die Gefahr der Reformierten spielte eine Rolle. Balthasar zählt auch den Umgang mit Kirchengütern auf, die „teils von den Weltlichen geraubt“ oder aber auch „sonst von ihrer Willkür und Gerichtsbar-

\footnotetext{
fassten Institutionen und auch kein Gesandtschaftsrecht. Aufgrund des mittelalterlichen Reisekönigtums gab es keine Hauptstadt, in der ständige Vertretungen eingerichtet werden konnten. In der Regel wurden Gesandte ad hoc und im zeitlich und inhaltlich eng begrenzten Auftrag entsandt oder aber es kam zu direkten Herrscherbegegnungen. Erst mit der Idee der „Staatsräson“ vollzog sich der Wandel vom Personenverbandsstaat des Mittelalters zum territorialen Flächenstaat der Neuzeit, der gleichzeitig mit einer De-Personalisierung der Herrschaft einherging.“
}

35 Vgl. Urban Fink: Die Luzerner Nuntiatur 1586-1873.

36 Die Schweizergarde wurde 1506 von Papst Julius II. eingeführt.

37 Ein wichtiges Pontifikat für die Beziehung zwischen Eidgenossenschaft und dem Papsttum war unter Gregor XIII. (1572-1585), auch wenn die in dieser Arbeit untersuchten Instruktionen und Berichte erst mit dem Pontifikat Sixtus V. ansetzen. Oswald Schön schreibt dazu: „Die Eidgenossenschaft blieb in zwei feindliche Theile zerspalten, von denen der eine an die päpstliche Oberhoheit gebunden war. Und wie sehr Rom mit den religiösen Interessen auch politische verband, beweist ein Breve, in welchem der Papst Gregor XIII. die Eidgenossen warnt, protestantische Orte in ihrem Bund aufzunehmen, mit den Worten: „Fern sei von Eurem Bunde der gottlose Genfer, der gottlose Rhätier!““”, zit. aus: Oswald Schön: Entwicklungsgeschichte der Schweizerischen Eidgenossenschaft, S. 178.

38 Zit. nach: Joseph Anton Balthasar: Helvetia, S. 84. 
keit“ verloren gingen. ${ }^{39}$ In seinem Werk zählt Balthasar die negativen Konsequenzen der Reformation auf, die dazu führten, dass Sixtus V. die Schweizer als „so unartig und unchristlich“ ansah. ${ }^{40}$

$\mathrm{Zu}$ Urban VII. gibt es nicht viel zu schreiben, da es sich um ein kurzes Pontifikat handelte. Beim Nachfolger Gregor XIV. ist es wieder Gardekommandant Sägisser zusammen mit dem Landammann von Unterwalden, Melchior Lussy, die im Namen der sieben katholischen Orte der Eidgenossenschaft gratulieren. ${ }^{41}$ Wiederum handelt es sich um ein kurzes Pontifikat, sodass wenig über den Bezug dieses Papstes zur Eidgenossenschaft gesagt werden kann. Dasselbe gilt dann für Innozenz IX. Mit Clemens VIII. hatte sich Lussy „länger unterhalten“. Es ging um die Schulden der römischen Kurie, was die Bezahlung der Söldner betraf. Doch schon Gregor XIV. ließ den Vertreter der katholischen Eidgenossenschaft diesbezüglich im Regen stehen. So schreibt Lussy 1591 aus Rom:

Wie dass Ihre Heiligkeit Gregor mit dem er der Länge nach Unterredung gepflogen, von der Bezahlung der Restanzen, die über 40000 Kronen betragen, nichts wissen wolle. Papst Sixtus habe nichts versprochen, und sich darüber öffentlich im Consistorio erklärt. $^{42}$

Der Papst ließ auch wissen, so fügt Lussy an, dass die Aufstände in der Eidgenossenschaft, die in Folge der fehlenden Einnahmen stattfanden, nicht auf „päpstlichen Geheiß“ geführt wurden, und deshalb liege es nicht am Papst, sondern am Anführer in der Eidgenossenschaft, die protestierenden Leute zu beruhigen..$^{43}$

Clemens VIII. hatte es weit schwerer mit den Schweizern. Das Verhältnis war vor allem durch ,juristische Streitigkeiten“ betrübt, sodass wegen der Gerichtsbarkeit „bei Verbrechen geistlicher Personen“, der Papst nicht bereit war, dies dem weltlichen Gericht zu überlassen. Die Konsequenz war, dass zwei Nuntien deswegen Luzern verlassen mussten. Dazu hatte Jahre später Nuntius Ranuccio Scotti geschrieben:

Die Hochachtung für den römischen Stuhl gibt dem Nuntius große Achtung; die Gesandtschaft ist aber schwierig, weil sie, um Einfluss zu erhalten, freigiebig sein muss, und nicht

39 Zit. nach: ebd., S. 84.

40 Zit. nach: ebd., S. 85. So wird aufgezählt, dass die zur reformierten Kirche konvertierten Priester die Kirchengüter gestohlen hätten und dass etliche Frauenklöster geschlossen worden seien.

41 Vgl. Ebd., S. 101.

42 Zit. nach: ebd., S. 101.

43 Vgl. ebd., S. 101. 
nur einen Fürsten oder Günstling, sondern so viele, die diese Orte regieren, zu gewinnen hat. $^{44}$

Mit Leo XI. gab es wiederum ein kurzes Pontifikat, dafür folgte mit Paul V. ein Nachfolger Petri, der fast zwei Jahrzehnte wirkte. Wie Balthasar festhält, wurde Jakob von Sonnenberg als Vertreter des Luzerner Rates nach Rom entsandt und zwar „im Namen der katholischen Orte“. In der Ewigen Stadt wurde er „zum Ritter geschlagen, so wie der ihn begleitende Tochtermann, Hauptmann Jost Kraft“. 45

Während dieses Pontifikats stach vor allem der Konkurrenzkampf zwischen dem Heiligen Stuhl und Venedig im Verhältnis zu den Eidgenossen als prägendes Element hervor. Vor allem Nuntius della Torre hatte über diesen Streit und die Sicht des Papstes klare Worte geäußert. So zitiert ihn Balthasar mit folgenden Worten:

Die Herren, sagt der Nuntius, werden es noch wohl in frischem Gedächtnis haben, was ich ihnen vor etwa zwei Monaten vorgetragen, und aus Befehl des heiligen Vaters communiciert, nämlich den schweren Handel, den er mit der Herrschaft Venedigs von wegen ihres trotzigen und halsstarrigen Ungehorsams, gegen den heiligen Römischen Stuhl hat, wo ich aus gutherzigem Gemüthe und getreuer Wohlmeinung, durch besondern Eifer zu der Herren Wohlfahrt, Lob, Ehre und Reputation, und um sie auch bei Ihre Heiligkeit in desto bessere Gnaden und Gunst für alle Zufälle zu erhalten, ihnen insinuiert und gerathen hatte, welcher Maßen sie sich wegen dieser Communication gegen den heiligen Stuhl schriftlich erklären und ihre Theilnahme erzeigen möchten, wie dann geschehen, und dasselbige Schreiben von mir den Eil an Ihr Heiligkeit überlassen worden. Und weil dann dieselbe sich gegen die Republik ganz väterlich und gnädig, durch ein herrliches und tröstliches Breve, das Ihr, wie billig zu Mehrung ihrer Reputation gereichen muß, erzeige, habe er nicht ermangeln wollen, besagtes Breve persönlich zu präsentieren. ${ }^{46}$

Auch im nachfolgenden Pontifikat von Gregor XV. spielte der Konflikt mit Venedig eine Rolle, allerdings ging es dabei vor allem um die Lage in Graubünden. Die drei Großmächte Venedig, Savoyen und Frankreich unterzeichneten am 7. Februar 1623 einen Vertrag, um gegen Spanien vorzugehen. Die Eidgenossenschaft wurde dazu eingeladen. Spanien und auch Österreich waren dagegen. Gregor XV. bot Madrid und Wien an, als Vermittler zu wirken, mit der Bitte, das Veltlin auszuschließen.

44 Zit. nach: Melchior Schuler: Die Thaten und Sitten der Eidgenossen, S. 156-157. Es ist nicht bekannt, wann dieser Brief von Scotti verfasst wurde.

45 Zit. nach: Joseph Anton Balthasar: Helvetia, S. 114.

46 Zit. nach: ebd., S. 144-115. 
Sein Wunsch, ließ er sich vernehmen, sei, Italien die Gräuel eines nochmaligen Krieges zu ersparen, und den Zwiespalt zwischen Söhnen der Kirche zu verhüten, welcher der Ketzerei nur zu neuer Erstarkung gereichen müßte. ${ }^{47}$

Daraufhin besetzte der „Papstbruder“, Orazio Ludovisi, Herzog von Fiano, das Veltlin mit einer päpstlichen Truppe. Doch Gregor XV. starb, und es folgte Urban VIII. und gab das Veltlin wieder zurück. Seine Bedingung war, dass das Veltlin Graubünden angeschlossen werden sollte. Frankreich fand die Idee alles andere als gut, und die Veltliner selber wollten lieber unter der Herrschaft des Heiligen Stuhls bleiben. 1648 wurde der Friedensvertrag zwischen Spanien und Frankreich unterzeichnet, doch der Nachfolger Urbans VIII., Papst Innozenz X., verweigerte im Hinblick auf die kirchlichen Zugeständnisse an die Protestanten seine Zustimmung. Mit dem Westfälischen Frieden von 1648 wurde die Eidgenossenschaft vom Reich unabhängig.

\subsubsection{Der italienische Kontext als Prägung der Nuntien}

Neben der persönlichen Biographie der einzelnen Nuntien spielte auch das soziokulturelle Umfeld bei ihrer Meinungsbildung eine Rolle. Deshalb ist die Geschichte Italiens des 16. und 17. Jahrhunderts für die Einordnung so wichtig. Einerseits stammten die meisten Nuntien aus eher wohlhabenden und noblen Familien, was ihre Einstellung gegenüber den Schweizer insofern prägte, als dass sie dort zwar einige wohlhabende Patrizierfamilien vorfanden, doch die gesellschaftspolitische Struktur, wie sie es von ihrer italienischen Heimat kannten, fehlte.

Italien in jener Zeit gab es als einheitlichen Staat noch nicht. Bekanntlich gab es viele größere und kleinere Staaten, die untereinander konkurrierten. Dennoch war im Denken jener Zeit das Konzept eines einheitlichen kulturellen und zum Teil auch sprachlichen Territoriums namens Italien durchaus präsent. ${ }^{48}$

Interessant ist auch, dass etliche Gesprächspartner in der Schweiz Italienisch sprachen. Wie Reutner und Schwarze hervorheben, war die italienische Sprache im deutschsprachigen Raum sehr verbreitet, denn „offensichtlich hat sich das Interesse in dieser Zeit v. a. auf die Kaufleute konzentriert, auf deren Druck dann Ende des 16. Jahrhunderts in den süddeutschen Handelsmetropo-

47 Zit. nach: Johann von Müller: Geschichten Schweizerischer Eidgenossenschaft während des 16. und 17. Jahrhunderts, S. 569.

48 Vgl. Peter Burschel: Das Eigene und das Fremde, S. 260-271. 
len Frankfurt und Ulm Italienischunterricht an höheren Schulen angeboten wird. “49

Dies zeigt auf, dass die Schweizer ebenfalls ihre „Gäste aus dem Süden“ als Italiener und nicht in erster Linie als Römer, Neapolitaner oder Lombarden betrachteten. Auch war die italienische Halbinsel in jener Zeit nicht unbedingt in einem Nord-Süd-Gefälle aufgeteilt, wie es in Italien seit spätestens Ende des Zweiten Weltkriegs gilt. Wie Galasso und Kellenbenz ${ }^{50}$ schreiben, sei Süditalien zwar ein wirtschaftlicher Außenposten Europas gewesen, der auch abhängig war, „aber nicht passiv“. So bleibe der wirtschaftliche Raum Süditaliens „für die gesamte Zeitspanne der mehr als zwei Jahrhunderte währenden Expansionsphase und der darauffolgenden Stagnationsphase von der zweiten Hälfte des 15. Jahrhunderts bis in das frühe 18. Jahrhundert einer der wichtigsten Pole für die Entstehung der modernen europäischen Wirtschaft"“. 51

Dass es trotz des Erbes des Römischen Reiches keinen einheitlichen Staat oder kein Staatsgebilde wie in Frankreich oder wie das Deutsche Reich gab, lag wohl an den vielen größeren und kleineren Hindernissen der Partikular-Interessen. Das Papsttum hätte der Kern eines italienischen Einheitsstaates oder Reiches sein können. Allein die Tatsache, dass der Kirchenstaat einen Großteil Mittelitaliens umfasste und als Referenzort aller auf der Halbinsel befindlichen Bischöfe galt, hätte für eine Einigung sorgen können. Doch die „partikularistischen Kräfte“ wie jene ,in der Toskana, im Raum von Ligurien und Piemont bis Venedig, im Königreich Neapel und auf den drei Inseln Sizilien, Sardinien und Korsika waren zu groß“. Hinzu kam, dass „ihre Machthaber sich erfolgreich den Bestrebungen der Einigung widersetzten“. Sie hatten sogar „den Versuchungen der großen auswärtigen Mächte nachgegeben, einzelne Teile dieses zersplitterten Italiens für ihre Interessen einer Vormachtpolitik zu nützen“. 52

Das politische Denken Italiens ${ }^{53}$ in jener Zeit war also geprägt von der Zähmung und Einschränkung - aus Sicht der italienischen Kleinstaaten und europäischen Mächten - der Macht der Päpste, ${ }^{54}$ und umgekehrt war es ein Anliegen der römischen Kurie, nicht nur ihre kirchliche, sondern auch ihre politischweltliche Macht $\mathrm{zu}$ festigen und auszubauen.

49 Zit. nach: Ursula Reutner, Sabine Schwarz: Geschichte der italienischen Sprache, S. 228.

50 Vgl. Hermann Kellenbenz: Finanzen und Staatsräson in der frühen Neuzeit Europas.

51 Zit. nach: Giuseppe Galasso: Wirtschaft und Finanzen im Mezzogiorno, S. 44.

52 Zit. nach: Hermann Kellenbenz: Finanzen und Staatsräson in der frühen Neuzeit Europas, S. 16.

53 Vgl. Oliver Hidalgo, Kai Nonnenmacher (Hrsg.): Die sprachliche Formierung der politischen Moderne, S. $22 \mathrm{f}$.

54 Man denke hierbei an das Traktat De potestate papae von 1537 von Philipp Melanchthon. 


\subsection{Die Nuntiatur}

Die Nuntiatur ist die päpstliche Gesandtschaft in einem Land. ${ }^{55}$ Seit der Einsetzung der päpstlichen Nuntiaturen wurden immer Geistliche - also zum Priester geweihte Männer - zu Nuntien ernannt. Dieser vertritt somit immer die „offizielle Stimme“ des Papstes in dem entsprechenden Gastland. Wenn man somit die Mentalität, Einstellung und Einschätzungen der Nuntien über das entsprechende Gastland untersuchen will, dann darf man dieses Element des „Priesterseins" nicht außer Acht lassen. ${ }^{56}$

Apostolische Nuntien mit ihren diplomatisch-geistlichen Aufgaben wurden in der Folge zu einer wichtigen Vermittlungsinstanz zwischen Rom und den lokalen Kirchen: Einerseits agierten sie als notwendige Referenten für die römische Kurie und als Vertreter für die Justizadministration, andererseits waren sie neben dem ortsansässigen Episkopat ein wichtiger Anlaufpunkt für die Gläubigen, um benötigte Gratien, Absolutionen, Dispense oder Lösungen von Konflikten zu erhalten. Sofern demnach die Reformbeschlüsse des Trienter Konzils betroffen waren, die einer Auslegung durch die Konzilskongregation bedurften, ist eine Kooperation sowie ein Kommunikationsnetzwerk entweder direkt zwischen den Nuntien und der Konzilskongregation und/oder über das Staatssekretariat anzunehmen. Auf diese Weise vermochte Rom über die Verwaltung der Nuntiaturen trotz der geographischen Distanz auf lokaler Ebene einzugreifen, um Informationen und Entscheidungen zur Lösung spezifischer Rechtsstreitigkeiten sowie von Fragen zu erhalten.

\subsubsection{Definition und Aufgabenbeschreibung der Nuntiatur im posttridentini- schen Kontext}

Da die katholische Kirche strukturell hierarchisch aufgebaut ist, ${ }^{57}$ zählt der Nuntius in dieser Skala „aus Rom“ betrachtet höher als ein Diözesanbischof, der die Ortskirche vertritt. Im Gegensatz zu einem Bischof hat der Nuntius vor

55 Vgl. http://www.hls-dhs-dss.ch/textes/d/D11742.php (31. Dezember 2020)

56 Es geht hier nicht um eine psychiatrische wissenschaftliche Betrachtung. Doch die persönliche Biographie sowie die „berufliche“ Rangordnung spielen bei einer solchen Untersuchung ohne Zweifel eine Rolle.

57 Dies kann man zumindest für das Papsttum ab dem 4. Jahrhundert behaupten. Für diese Untersuchung behandeln wir jene Zeit der Kirchengeschichte, in der sich das Verhältnis zwischen Papsttum und Kaisertum in Europa bereits etabliert hatte und beide in einem relativ stabilen Verhältnis miteinander auskamen. 
allem eine „diplomatische Funktion“. ${ }^{58}$ Das galt bereits für die Nuntiaturen des 16. Jahrhunderts, während ein Bischof vor allem eine „pastorale Aufgabe“ wahrnimmt. Die Bedeutung eines Gesandten des Papstes außerhalb Roms ist historisch vor allem aufgrund der „Kontrolle“ über die Ortskirchen verbunden. ${ }^{59}$

Die Aufgabe der Nuntien als Überwacher und Förderer des innerkirchlichen Reformprogramms wurde mit dem Pontifikat von Gregor XIII. in Europa übernommen. Hierbei erhielten die Nuntien als Grundlage für ihr Handeln vom Staatssekretariat beschriebene Instruktionen (Weisungen) und Fakultäten (geistliche Vollmachten). Diese galten sowohl auf diplomatischer als auch auf kirchlicher Ebene. So oblag dem päpstlichen Vertreter neben seinen diplomatischen Aufträgen zum Beispiel die Abhaltung von Visitationen der Bistümer und Klöster, die Bestellung geeigneter Personen zu Bischöfen, Kontrolle und Unterstützung des örtlichen Episkopats, Förderung des Priesternachwuchses, Kontrolle des Büchermarktes und anderes mehr.

\subsubsection{Die Auswahlkriterien für die Nuntiaturstelle}

Als Bischöfe von Rom verstanden sich die Päpste spätestens ab dem 11. Jahrhundert mit der sogenannten „Gregorianischen Reform“60 immer mehr als „Kirchenoberhäupter“ und weniger als „Primus inter pares“, wie es hingegen die Patriarchen der Ostkirchen kannten. Die Sendung von Legaten und Gesandten, die spätestens ab dem 16. Jahrhundert auch Nuntien genannt wurden, hatte somit die Funktion der „Erforschung der Höfe, der Völker und des Klerus“ und „erleichterte“ auf diese Weise die Leitung der katholischen Kirche von oben herab. ${ }^{61}$

58 Vgl. Christian Wilhelm von Glück, Ludwig Snell: Geschichte der Einführung in die Schweiz und ihre dargelegte Politik.

59 Vgl. Alexander Koller: Einige Bemerkungen zum Karriereverlauf der päpstlichen Nuntien am Kaiserhof (1559-1655), S. 841: „Die Nuntiaturen zählen seit Beginn der Neuzeit zu den herausragenden und am meisten wahrgenommenen Ämtern der römischen Kurie.“

60 Papst Gregor VII. (Pontifikat von 1073 bis 1085) beanspruchte in seinem „Dictatus papae“ von 1074 den Titel und die Jurisdiktion eines „Universalbischofs“ exklusiv. Die Amtszeit dieses Papstes mündete in den sogenannten Investiturstreit, der für das Reich erst im Wormser Konkordat von 1122 beigelegt wurde. 1075 ließ Gregor VII. 27 Leitsätze, den „Dictatus Papae“, aufzeichnen, in denen er unter anderem die Vorrangstellung der geistlichen Gewalt gegenüber weltlichen Machthabern betonte. Vgl. Rudolf Schieffer: Papst Gregor VII. Kirchenreform und Investiturstreit.

61 Vgl. Leopold von Ranke: Die römischen Päpste. 
Das Besondere an der Nuntiatur in der Eidgenossenschaft bestand zu Beginn des 16. Jahrhunderts in zweierlei Hinsicht: So waren die Papst-Gesandten mit politischen Aufgaben betraut. Dazu zählte vor allem die Anwerbung von Söldnern, was gleichzeitig auch eine ökonomische Bedeutung vor allem für die betroffenen Schweizer Familien hatte. Das zweite Element war die kirchenrechtlich-lehramtliche Aufgabe der Nuntien. Es war eine der zentralen Aufgaben der Nuntien, die „Reformen des Konzils von Trient“ in der Eidgenossenschaft umzusetzen und zu „prüfen“, ob sich der Klerus und das Kirchenvolk auch an die „Regeln des Konzils“ hielten. Deshalb nahmen die Nuntien auch eine „quasibischöfliche“ Funktion ein, da sie sich pastoral engagieren mussten, was eigentlich die Aufgabe eines Bischofs ist. ${ }^{62}$

In gewisser Weise gilt dies heute noch. Der Nuntius stand auch früher - so wie es heute auch der Fall ist - immer in direktem Kontakt mit dem Staatsekretariat in Rom, am Sitz des Papstes. Für Historiker ist somit der Schriftverkehr zwischen der Nuntiatur und dem römischen Staatssekretariat von besonderer Bedeutung. ${ }^{63}$

Nuntien vertreten also die Interessen des Papstes und sind für den politischen - früher auch ökonomischen und teils militärischen - Austausch mit staatlichen Institutionen und Einrichtungen verantwortlich. ${ }^{64}$

Der dritte wichtige Aspekt der Nuntiatur bestand in ihrer Funktion als „Beobachter“ und „Agent“ gegen die reformatorischen Kräfte nördlich der Alpen. ${ }^{65}$ Da gerade der geographische Raum der heutigen Schweiz eines der zentralen Gebiete der Reformation war, wurde aus päpstlicher Sicht jenem Gebiet eine besondere Rolle zugeschrieben. Der Papst wurde jedoch umgekehrt zur Zielscheibe der Reformatoren, und das führte „gezwungenermaßen“ $\mathrm{zu}$ einer entgegengesetzten Strategie, um diese Angriffe zumindest wahrzunehmen und im besten Falle auch entgegenzuwirken. ${ }^{66}$

62 Vgl. Michael F. Feldkamp: Die europäischen Nuntiaturen in der frühen Neuzeit.

63 Vgl. Pierre Blet: Histoire de la Représentation Diplomatique du Saint Siège.

64 Das galt sowohl im 16. Jahrhundert und das gilt auch heute. Die Nuntiatur in unserer Zeit ist von den Richtlinien und Vorgaben von Papst Paul VI. (1963-1978) geprägt. Unter den Aufgaben eines Nuntius ist heute die wichtigste die, das „Band der Einheit“ zwischen der katholischen Kirche in diesem Land und der Weltkirche zu stärken, wie es in der entsprechenden Bestimmung von Papst Paul VI. aus dem Jahr 1969 heißt. Vgl. Michael F. Feldkamp: Die europäischen Nuntiaturen in der frühen Neuzeit.

65 Vgl. Hubert Jedin: Geschichte des Konzils von Trient, Bd. 3, S. 7: „Das Konzil brauchte, um $\mathrm{zu}$ funktionieren, einen Beamtenapparat.“

66 Vgl. František Šmahel (Hrsg.): Häresie und vorzeitige Reformation im Spätmittelalter. 


\subsubsection{Das Arbeitsumfeld der Nuntien}

Die Nuntiaturen ab dem 16. Jahrhundert waren in zwei Kategorien zu unterscheiden: Jene, die auf der italienischen Halbinsel waren, zählten einerseits durch die geographische Nähe zu Rom und andererseits durch die „näheren“ kulturellen und sprachlichen Komponenten als beliebte Stellen, die der Karriere der Amtsträger sicherlich auch als förderlich erachtet wurden. Die zweite Kategorie von Nuntiaturen betraf hingegen jene, die außerhalb Italiens lagen. Dort mussten sie sich gezwungenermaßen auf „fremde Lebenswelten einlassen“ und auf diese Weise „besonderen Herausforderungen stellen“. Doch auch ausländische Nuntiaturstellen konnten durchaus als Karrieresprungbrett betrachtet werden, denn sie besaßen „eine umso größere Bedeutung für die Kurie durch ihre Beobachtungen und Informationsakquise auf wenig vertrautem Terrain“. ${ }^{67}$

Die Schweiz war im Grunde geographisch nicht weit vom Zentrum entfernt, zumindest nicht viel weiter als Mailand und sicherlich näher als Köln, Wien, Paris oder Lissabon, und trotzdem ,fühlten sich die Nuntien potenziert mit Andersartigkeit konfrontiert“. ${ }^{68}$ Dennoch wurde dies „für die römische Kurie jedoch eben auch“ als „,besondere Erkenntnismöglichkeit“ betrachtet. ${ }^{69}$ Das ausgebaute Nuntiaturnetz konkurrierte mit den Botschaftern Venedigs, die jedoch eine vorwiegend wirtschaftliche Bedeutung innehatten und dem Handel und den militärischen Interessen der Lagunenstadt dienten. ${ }^{70}$

Einen Vorteil im Arbeitsumfeld der Nuntiatur in der Eidgenossenschaft im Gegensatz zu jenen am Kaiserhof oder in Paris war die Tatsache, dass das zuständige Territorium kleiner und kompakter war. Die anderen bedeutenden Nuntiaturen hatten „einen riesigen Sprengel zu beaufsichtigen“ ${ }^{71}$ Eine weitere Besonderheit, die eine Nuntiaturstelle im deutschen Sprachraum betraf, lag an der Tatsache, dass sie sich in unmittelbarer Nähe zum „Feind“ - also zur protestantischen Welt - befand. Es gab diesbezüglich aber nur indirekte und informelle Kontakte. Hier spielten die Kontakte $\mathrm{zu}$ den Botschaftern Frankreichs

67 Zit. nach: Guido Braun: Akteure, Medien und Institutionen in den Prozessen von Wissensproduktion über das Reich an der römischen Kurie in den 1620er Jahren, S. 213. Mit „Informationsakquise" ist die Informationsbeschaffung gemeint.

68 Zit. nach: ebd., S. 213.

69 Ebd., S. 213.

70 Mit dem Frieden von Lodi 1454 erfolgte eine vorläufige Grenzziehung des Territoriums Venedigs. Die Adda wurde als venezianische Westgrenze festgelegt. Diese Eroberungen und mehrere Versuche, Ferrara, auf das der Kirchenstaat Anspruch erhob, zu erobern, führten dazu, dass der Kirchenstaat und die meisten anderen italienischen Staaten nun in Venedig ihren schärfsten Rivalen sahen.

71 Zit. nach: ebd., S. 214. 
oder Venedigs eine wichtige Rolle, „um Neuigkeiten über die politischen Entwicklungen“ $z u$ erfahren. ${ }^{72}$

Um sich in einem Land zu orientieren, standen den Nuntien die Diplomatenspiegel zur Verfügung. Darin waren Literaturempfehlungen enthalten. Auch in vielen Instruktionen wurden Geschichtswerke aufgelistet, die zur Lektüre und zum Studium des Gastlandes empfohlen wurden. ${ }^{73}$

\subsubsection{Die grundlegenden Aufgaben der Nuntien als Diplomaten: Repräsentie- ren, Informieren und Verhandeln}

Hatten die Nuntien im 16. und 17. Jahrhundert vor allem eine Funktion, die vom Konzil von Trient und den Bestrebungen der „katholischen Reform“ ausgingen, so waren die Gesandten an den ständigen Nuntiaturstellen durchaus Diplomaten im juristischen Sinne. Mit der Reform von Papst Gregor XIII. wurden die Aufgaben des Repräsentierens gleichgestellt mit Informieren und Verhandeln. ${ }^{74}$ Die Nuntien in Luzern haben den Papst im kirchlichen Bereich nicht nur bei Visitationen in Klöstern oder Pfarreien vertreten, auch bei liturgischen Anlässen sowie bei Sakramentenspendungen waren sie vertreten. Hier waren sie aber eher zurückhaltend, um - wie sie in Briefen schreiben - nicht dem sakramentalen Bereich der lokalen Bischöfe im Weg zu stehen. Die Informationsbeschaffung hingegen wurde durch persönliche Kontakte gepflegt, die auch namentlich als Quellen in den chiffrierten Briefen genannt werden. Das Informationsnetzwerk wurde auch bei Verhandlungen eingesetzt, da die Nuntien aus sprachlichen oder opportunistischen Gründen nicht selber an Auseinandersetzungen teilnehmen konnten. ${ }^{75}$

Die Nuntien in Luzern haben in der Phase, die dem Mailänder Kardinal Borromäus zu verdanken ist, vor allem durch Visitationen ihr Amt ausgeübt. ${ }^{76}$ Das gilt zumindest für die Zeit des 16. Jahrhunderts und zu Beginn des 17. Jahrhun-

72 Vgl. ebd., S. 214.

73 Vgl. Leo Just: Die Erforschung der päpstlichen Nuntiaturen.

74 Vgl. Grégoire XIII, l'empire et l'évolution de la nonciature posttridentine (1572-1585), S. 8998.

75 Vgl. I viaggi dei nunzi in area germanica, S. 91-110.

76 Vgl. Hubert Jedin: Geschichte des Konzils, Bd. 3, S. 4: „Was in Trient über die Kirchenreform beschlossen und seitdem in der Kirche durchgeführt worden war, das Werk des hl. Karl Borromäus, das Werk der Pius, Gregor und Sixtus, alles galt ihm nichts, weil Staatskirchentum und Episkopalismus in Trient nicht gesiegt hatten, vielmehr das Papsttum die Trienter Dekrete zum Palladium der katholischen Reform wie der Gegenreformation gemacht, durch sie neuen Einfluß und neues Ansehen gewonnen hatte.“ 
derts. Auf diese Weise hatten sie die Möglichkeit, sich direkt ein Bild von der Lage vor Ort zu machen. Sie waren aber vor allem auf der Suche nach Missständen und Möglichkeiten, das religiöse Leben zu verbessern oder zumindest $\mathrm{zu}$ fördern. Wie in der Korrespondenz nachzulesen ist, nahmen die Gastgeber - in diesem Falle die Luzerner - die Nuntien positiv auf. Zwar berichten Historiker von anfänglich ablehnenden Haltungen der „Politiker, die um ihre staatskirchenrechtlichen Privilegien fürchteten“, ${ }^{77}$ doch dies kommt in der Korrespondenz der Nuntien nach Rom kaum vor und wenn, dann nur um aufzuzeigen, dass die Reformbestrebungen aus Rom wichtig seien.

\subsubsection{Die spezifischen Rahmenbedingungen für die Nuntien in der Eidgenos- senschaft}

Die nach Luzern gesandten Bischöfe mussten sich auf ihre Reise vorbereiten. Dazu gehörten auch die Finanzierungsfrage sowie das Organisieren des Haushalts. In den Instruktionen wird darauf ausgiebig eingegangen. Die Reform des Nuntiaturwesens und damit die Einsetzung einer ständigen Nuntiatur in Luzern brachte es mit sich, dass von der Reise über den Umgang bis hin zum alltäglichen Aufenthalt nicht nur vieles von den Instruktionen vorgegeben und geregelt wurde, sondern sie auch eine Starthilfe für die neuen Nuntien waren. ${ }^{78}$

Der konkrete Alltag der Nuntien in Luzern wird anschaulich von Fink dargestellt. ${ }^{79}$ In den Briefen aus Luzern berichten die Nuntien viel über den Alltag. Es geht um Beobachtungen politischer und sozialer sowie auch kirchenpolitischer Entwicklungen. In den Briefen werden selten auch finanzielle Nöte der Nuntien behandelt. Sie konnten als Diözesanbischöfe mit den Einnahmen aus ihren Bistümern rechnen. ${ }^{80}$ Der Aufenthalt war nicht sonderlich günstig, aber das Vorurteil, das man heutzutage gegenüber der „teuren“ Schweiz hegt, ist in den Briefen aus dem 16. und 17. Jahrhundert nicht $\mathrm{zu}$ finden. Vielmehr liest man davon, dass Luzern und allgemein die Eidgenossenschaft als Provinz zu betrachten ist. Üblich war, wie es Biaudet beschreibt, dass die Nuntien im 16. Jahrhundert entweder eine festgesetzte Summe vor ihrer Abreise bekamen, um damit im Gastland zu leben, oder eine monatliche Finanzhilfe (,imdemnité"). ${ }^{81}$ In den Briefen und Instruktionen für die Nuntien in Luzern wird über Geldange-

77 Zit. nach: Lukas Vischer, Lukas Schenker, Rudolf Dellsperger (Hrsg.): Ökumenische Kirchengeschichte der Schweiz, S. 152.

78 Vgl. Pius Hafner: Staat und Kirche im Kanton Luzern.

79 Vgl. Urban Fink: Die Luzerner Nuntiatur 1586-1873.

$80 \mathrm{Vgl}$. Henri Biaudet: Les nonciatures apostoliques permanentes jusqu'en 1648.

81 Vgl. ebd., S. 68. 
legenheiten nicht direkt gesprochen. Ein damit verbundenes Problem war auch die Währungsfrage. Jegliche wirtschaftliche Angelegenheiten wurden in „scudi“ (,scudo d'oro“) angegeben. Wie Biaudet weiter unterstreicht, kam es auch auf die Protégés der Nuntien an. Diesen konnten sie finanziell und logistisch unter die Arme greifen, was bei den meisten Nuntien in Luzern der Fall war. Wurde die Stadt am Vierwaldstättersee als „provinziell“ betrachtet, so haben sie gleichzeitig ihr Amt in der Eidgenossenschaft als Karrierestelle betrachtet, um nach dem Ende ihrer Amtszeit die „Gewinne“ einzutreiben, indem sie eine „bessere" Stelle oder eine besondere kirchliche Ernennung als Gegenleistung erhielten. ${ }^{82}$

Eine ständige Nuntiatur bedeutete einen nicht unwesentlichen Kostenaufwand, einmal in der Bezahlung des Gesandten, aber vor allem durch die Nachrichtenverbindung, die man aus Sicherheitsgründen selbst besorgen musste. Das galt auch für den Apostolischen Stuhl, der einen hoch entwickelten Chiffrierwesen benutzte. Wie in vielen anderen Ländern musste sich der Nuntius in der Eidgenossenschaft auch mit der Abneigung gegen die ständigen Gesandten eines fremden Landes und Kultur auseinandersetzen. Gerade die protestantischen Schweizer betrachtete die Nuntien als Spione oder wenigstens als unerwünschte, dauernd fließende Informationsquelle für das Papsttum.

82 Vgl. ebd., S. 75. 